\begin{tabular}{|c|c|c|}
\hline 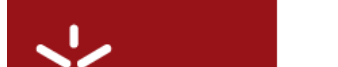 & CMAT & 1 \\
\hline - Reprositónill & Centro de Matemática da Universidade do Minho & -0 \\
\hline \multirow[t]{2}{*}{ Universidade do Minho } & Campus de Gualtar $4710-057$ Braga Portugal & $\begin{array}{l}\text { Universidade do Minho } \\
\text { Escola de Ciências }\end{array}$ \\
\hline & www.cmat.uminho.pt & Centro de Matemática \\
\hline
\end{tabular}

\title{
A two obstacles coupled problem
}

\author{
Assis Azevedo \\ Lisa Santos
}

CMAT and DMA, Universidade do Minho, Portugal

\section{Information}

Keywords:

Coupled problem

Variational inequality

Two obstacles

Original publication:

Nonlinear Analysis 120 (2015) 107 -

117, DOI:10.1016/j.na.2015.02.019

www.elsevier.com

\begin{abstract}
We consider a system of an evolutionary variational inequality of two obstacles type, depending on the temperature, coupled with the heat equation. We prove existence of solution of this system and we present examples that motivated this work. In particular, with additional assumptions on the data, we prove that solutions of this problem are also solutions of a similar problem where the convex set is of gradient constraint type (that depends on the temperature), improving a previous result.
\end{abstract}

\section{Introduction}

In certain situations, there exists a close relation between a variational inequality with gradient constraint and the same variational inequality with two obstacles. The following well-known variational formulation of the elastic-plastic torsion problem: to find $u \in \mathbb{K}^{\nabla}=\left\{v \in H_{0}^{1}(\Omega):|\nabla v| \leq 1\right\}$ such that, for $\beta$ a positive constant,

$$
\int_{\Omega} \nabla u \cdot \nabla(v-u) \geq \beta \int_{\Omega}(v-u) \quad \forall v \in \mathbb{K}^{\nabla}
$$

is known to be equivalent to the same variational inequality with the convex set $\mathbb{K}^{\nabla}$ replaced by

$$
\mathbb{K}=\left\{v \in H_{0}^{1}(\Omega):-d(\cdot, \partial \Omega) \leq v \leq d(\cdot, \partial \Omega)\right\}
$$

where $d$ denotes the euclidean distance. For details about the above results see [2, 3, 4], among others.

These remarks can easily be extended to the evolutionary case (for which the existence of solution is not so obvious). The solution $u$, belonging to a suitable space, such that $u(t) \in \mathbb{K}^{\nabla}, u(0)=u_{0} \in \mathbb{K}^{\nabla}$ and

$$
\int_{\Omega} \partial_{t} u(t)(v-u(t))+\int_{\Omega} \nabla u(t) \cdot \nabla(v-u(t)) \geq \beta(t) \int_{\Omega}(v-u(t)) \quad \forall v \in \mathbb{K}^{\nabla}, \text { for a.e. } t \in(0, T),
$$

is also the solution of the same variational inequality with convex set $\mathbb{K}$. Here, $\partial_{t}$ denotes the partial derivative with respect to the variable $t$ and $\nabla=\left(\partial_{x_{1}}, \ldots, \partial_{x_{d}}\right)$ is the spatial gradient. 
There exists a commitment between the two approaches. It is easier to prove existence of solution of the two obstacles problem but, on the other hand, the solution of the problem with gradient constraint is immediately more regular, more precisely, it belongs to $L^{\infty}\left(0, T ; W^{1, \infty}(\Omega)\right)$. So, it may be relevant to prove that the same function solves the two problems in more general situations.

Several physical problems are modelled using variational formulations with constraints on the first derivatives of the solutions. Examples can be found, for instance, in the papers $[12,11,7,13]$.

An interesting model consists of a system of an electromagnetic variational inequality, where the curl of the magnetic field is constrained by a function of the temperature, which is solution of the heat equation with source term depending on the magnetic field. Considering $\Omega$ a bounded open subset of $\mathbb{R}^{3}$ and denoting by $Q$ the cylinder $\Omega \times(0, T)$ and by $\Sigma$ the lateral surface $\partial \Omega \times(0, T)$, the authors solved, in [1], a simplified version of this model, by considering a longitudinal geometry, more precisely, assuming that the magnetic field $\boldsymbol{h}=(0,0, u)$. So, $\nabla \times \boldsymbol{h}=\left(\partial_{x_{2}} u,-\partial_{x_{1}} u, 0\right)$, and we arrive at the following coupled problem with gradient constraint (for details see [1]): to find $(u, \theta)$ belonging to a convenient space such that

$$
\begin{aligned}
& \left\{\begin{array}{r}
u(t) \in \mathbb{K}_{F(\theta(t))}^{\nabla} \text { for a.e. } t \in(0, T), \quad u(0)=u_{0} \in \mathbb{K}_{F\left(\theta_{0}\right)}^{\nabla} \\
\int_{\Omega} \partial_{t} u(t)(v-u(t))+\int_{\Omega} \nabla u(t) \cdot \nabla(v-u(t)) \geq \int_{Q} f(t)(v-u(t)) \\
\forall v \in \mathbb{K}_{F(\theta(t))}^{\nabla}, \text { for a.e. } t \in(0, T)
\end{array}\right. \\
& \left\{\begin{array}{l}
\partial_{t} \theta-\triangle \theta=g(u) \text { in } Q \\
\theta=0 \text { on } \Sigma \\
\theta(0)=\theta_{0} \text { on } \Omega,
\end{array}\right.
\end{aligned}
$$

where $f: Q \rightarrow \mathbb{R}, g: \mathbb{R} \rightarrow \mathbb{R}$ and $F: \mathbb{R} \rightarrow \mathbb{R}^{+}$are given functions and

$$
\mathbb{K}_{F(\theta(t))}^{\nabla}=\left\{v \in H_{0}^{1}(\Omega):|\nabla v| \leq F(\theta(t)) \text { a.e. in } \Omega\right\}
$$

A natural way to solve evolutionary variational inequalities consists of approximating them by a family of penalized equations. As far as the authors know, the only type of explicit regular penalization of a variational inequality with gradient constraint is performed by a family of quasilinear parabolic equations. As a consequence of this penalization and of the coupling, the a priori estimate of the time derivative of the solution of the variational inequality is obtained only in $\left(L^{\infty}\left(0, T ; W_{\infty, 0}^{1}(\Omega)\right)\right)^{\prime}$ and the existence result for the coupled problem treated in [1] does not correspond to a strong variational formulation. We remark that the variational inequality is, in fact, quasi-variational, as the convex set depends on $\theta$, solution of the heat equation, which depends on $u$. To treat this kind of problems, there exist other possible approaches, for instance semigroup theory, as in the one-dimensional quasi-variational evolutionary problem in [5] or time dependent subdifferential theory, as in [6].

At least for special classes of data, we are able to prove that a solution of a two obstacles coupled problem also solves the gradient constraint coupled problem, improving the "regularity" result obtained in [1] for the time derivative of this solution and obtaining existence for the strong variational formulation.

Although the main motivation for this paper is the one indicated above, it is worthwhile to prove existence of solution of the following more general two obstacles coupled system: given functions $f: Q \rightarrow \mathbb{R}$ and 
$g: \mathbb{R} \rightarrow \mathbb{R}$, operators $A, B$ with domain conveniently defined and the convex set

$$
\mathbb{K}_{A \theta(t)}^{B \theta(t)}=\left\{v \in H_{0}^{1}(\Omega): A \theta(t) \leq v \leq B \theta(t) \text { a.e. in } \Omega\right\}
$$

to find $(u, \theta)$ in a convenient space, solving the system (4)-(2), where

$$
\left\{\begin{array}{r}
u(t) \in \mathbb{K}_{A \theta(t)}^{B \theta(t)} \text { for a.e. } t \in(0, T), \quad u(0)=u_{0} \in \mathbb{K}_{A \theta_{0}}^{B \theta_{0}} \\
\int_{\Omega} \partial_{t} u(t)(v-u(t))+\int_{\Omega} \nabla u(t) \cdot \nabla(v-u(t)) \geq \int_{Q} f(t)(v-u(t)) \\
\forall v \in \mathbb{K}_{A \theta(t)}^{B \theta(t)}, \text { for a.e. } t \in(0, T) .
\end{array}\right.
$$

In Section 2 we present two examples of special choices for the operators $A$ and $B$, a theorem of existence and regularity of the solutions of problem (4)-(2) and a theorem of existence of regular solutions of problem (1)-(2), with additional assumptions on the data. The proof of this last theorem will use the results obtained for the two obstacles system.

Section 3 is concerned with the proof of existence of solution of a coupled penalized problem that approximates the two obstacles coupled problem, based on obtaining a priori estimates and applying Schauder fixed point theorem. We also prove the first theorem of Section 2, more precisely, existence of solution of problem (4)-(2), as well as the existence of solution for the operators $A$ and $B$ chosen in the first example.

In Section 4 we prove existence of solution for the problem with operators $A$ and $B$ given by the second example. Besides, we prove that the solutions of this last problem solve the gradient constraint problem (1)-(2), if suitable assumptions are imposed on the data.

Relevant open questions for the coupled two obstacles problem are the uniqueness of solution as well as its asymptotic behaviour in time.

In this paper we use, for instance, the notation $W_{p}^{2,1}(Q)$ for the Sobolev space $W_{p}^{1}\left(0, T ; L^{p}(\Omega)\right) \cap$ $L^{p}\left(0, T ; W_{p}^{2}(\Omega)\right)$ and, for consistency of notations, we use $W_{p}^{1}(\Omega)$ and $W_{p, 0}^{1}(\Omega)$ instead of $W^{1, p}(\Omega)$ and $W_{0}^{1, p}(\Omega)$, respectively.

\section{Main results}

Let $I$ be an interval of $\mathbb{R}$ and consider two operators $A, B: C(\bar{Q} ; I) \rightarrow C(\bar{Q} ; \mathbb{R})$ and, for a given function $\theta \in C(\bar{Q} ; I)$ and $t \in[0, T]$

$$
\mathbb{K}_{A \theta(t)}^{B \theta(t)}=\left\{v \in H_{0}^{1}(\Omega): A \theta(t) \leq v \leq B \theta(t) \text { a.e. in } \Omega\right\}
$$

This definition has also a sense if $\theta: \bar{\Omega} \rightarrow I$, as long as we consider $\theta$ as a function from $\bar{Q}$ into $I$, constant in the variable $t$.

We start by presenting two examples of possible choices for the operators $A$ and $B$. The second example will enable us to relate the solutions of the double obstacle system with the system with gradient constraint.

Example 2.1. Fix an interval $I$ of $\mathbb{R}$ and let $\varphi, \psi \in C(I ; \mathbb{R})$ be such that $\varphi<\psi$ and $\varphi(0) \leq 0 \leq \psi(0)$. Define, for $\theta \in C(\bar{Q} ; I)$,

$$
A \theta=\varphi \circ \theta \quad \text { and } \quad B \theta=\psi \circ \theta
$$


Example 2.2. Fix the interval I equal to $\mathbb{R}$. Given $F \in C\left(\mathbb{R} ; \mathbb{R}^{+}\right)$, if $\mathbb{K}_{F(\theta(t))}^{\nabla}$ is the convex set defined in (3), let

$$
A \theta(t)=\bigwedge_{v \in \mathbb{K}_{F(\theta(t))}^{\nabla}} v \quad \text { and } \quad B \theta(t)=\bigvee_{v \in \mathbb{K}_{F(\theta(t))}^{\nabla}} v .
$$

We start by fixing some assumptions on the data. Let $f: Q \rightarrow \mathbb{R}, g: \mathbb{R} \rightarrow \mathbb{R}, u_{0}, \theta_{0}: \Omega \rightarrow \mathbb{R}$ and assume that, for given $p, q>d$,

$$
\begin{gathered}
g \in C(\mathbb{R}) \quad \text { and } \quad \theta_{0} \in W_{q}^{2}(\Omega) \cap W_{q, 0}^{1}(\Omega) \\
f \in L^{\infty}(Q) \quad \text { and } \quad u_{0} \in \mathbb{K}_{A \theta_{0}}^{B \theta_{0}} \cap W_{p}^{2}(\Omega)
\end{gathered}
$$

$A, B$ continuous, $\quad A<B, \quad A 0 \leq 0 \leq B 0$.

Theorem 2.3. Assume (5)-(7) and the existence of nonnegative constants $a_{0}$ and $a_{1}$ such that

$$
\exists r \in[0,1) \forall s \in \mathbb{R} \quad|g(s)| \leq a_{0}|s|^{r}+a_{1} .
$$

Suppose in addition that if $\theta \in W_{q}^{2,1}(Q)$ then $A \theta, B \theta \in W_{q}^{2,1}(Q)$ and,

$$
\partial_{t}(A \theta)-\Delta(A \theta) \leq c\left|\partial_{t} \theta-\Delta \theta\right|, \quad \partial_{t}(B \theta)-\Delta(B \theta) \geq-c\left|\partial_{t} \theta-\Delta \theta\right|,
$$

being $c$ a positive constant.

Then problem (4)-(2) has a solution $(u, \theta) \in W_{p}^{2,1}(Q) \times W_{q}^{2,1}(Q)$.

Corollary 2.4. Assume (5) and (6). For an interval I depending only on the given data, let $A, B$ be defined as in Example 2.1. Assume, in addition, that

$$
\varphi, \psi \in W_{\infty}^{1}(I ; \mathbb{R}) \cap W_{1}^{2}(I ; \mathbb{R}), \quad \varphi^{\prime \prime} \geq 0 \quad \text { and } \quad \psi^{\prime \prime} \leq 0 .
$$

Then problem (4)-(2) has a solution $(u, \theta) \in W_{p}^{2,1}(Q) \times W_{q}^{2,1}(Q)$.

We say that a function $g: \mathbb{R} \rightarrow \mathbb{R}$ is hölderian with exponent $\beta, 0<\beta \leq 1$ if there exists a positive constant $M$ such that $|g(x)-g(y)| \leq M|x-y|^{\beta}$, for all $x, y \in \mathbb{R}$. We will denote this class of functions by $C_{\beta}^{0}(\mathbb{R})$. Observe that, if $g \in C_{\beta}^{0}(\mathbb{R})$ then it satisfies the growth condition

$$
|g(s)| \leq M|s|^{\beta}+g(0), \quad \forall s \in \mathbb{R},
$$

but the reverse is not true.

Theorem 2.5. Let $F \in C^{2}(\mathbb{R})$ be such that $0<F_{*} \leq F \leq F^{*}$ and $A, B$ be defined as in Example 2.2. Assume (6) and, for $p>d, \alpha=1-d / p$ and $0<\beta \leq 1$,

$$
g \in C_{\beta}^{0}(\mathbb{R}) \quad \text { and } \quad \theta_{0} \in C_{\alpha \beta}^{2}(\bar{\Omega}) .
$$

Then problem (4)-(2) has a solution $(u, \theta) \in W_{p}^{2,1}(Q) \times C_{\alpha \beta, \alpha \beta / 2}^{2,1}(\bar{Q})$.

Assume in addition that: (i) $f$ does not depend on $x$; (ii) $F^{2}$ is convex; (iii) $F$ is decreasing and $g$ nonnegative or $F$ is increasing and $g$ nonpositive; (iv) $u_{0} \in \mathbb{K}_{F(\theta(t))}^{\nabla}$.

Then problem (1)-(2) has a solution $(u, \theta) \in W_{p}^{2,1}(\Omega) \times C_{\alpha \beta, \alpha \beta / 2}^{2,1}(\bar{Q})$.

Remark 2.6. In this last theorem, the stronger assumptions on $g$ and $\theta_{0}$ are needed to study the regularity of the particular type of obstacles we are considering (see Proposition 4.2). 


\section{Existence of solution of the coupled two obstacles system}

The proof of existence of solution of problem (4)-(2) will be done approximating this system by a family of coupled systems of evolutionary equations. Existence of solution for the decoupled equations is known. The application of a fixed point theorem, after proving the continuous dependence on the data, together with obtaining a priori estimates for the approximating solutions, will allow us to find a solution of our problem as a limit of solutions of the approximating problems. The growth condition (8) on $g$, in Theorem 2.3, will only be needed to pass to the limit.

\subsection{The penalized problem}

In this section $\varepsilon$ is a small positive number and $m$ a large positive number that will be chosen in the proof of Proposition 3.7. Let $\eta_{\varepsilon}: \mathbb{R} \rightarrow \mathbb{R}$ be a smooth nondecreasing function such that $\eta_{\varepsilon}(s)=0$ for $s \geq 0$ and $\eta_{\varepsilon}(s)=-1$ for $s \leq-\varepsilon$ and consider the penalized and regularized problem

$$
\left\{\begin{array}{l}
\partial_{t} u^{\varepsilon}-\triangle u^{\varepsilon}+m \eta_{\varepsilon}\left(u^{\varepsilon}-A \theta^{\varepsilon}\right)-m \eta_{\varepsilon}\left(B \theta^{\varepsilon}-u^{\varepsilon}\right)=f^{\varepsilon} \quad \text { in } Q \\
u^{\varepsilon}(0)=u_{0}^{\varepsilon} \text { on } \Omega, \quad u_{\mid \Sigma}^{\varepsilon}=0 \\
\partial_{t} \theta^{\varepsilon}-\triangle \theta^{\varepsilon}=g^{\varepsilon}\left(u^{\varepsilon}\right) \quad \text { in } Q \\
\theta^{\varepsilon}(0)=\theta_{0}^{\varepsilon} \text { on } \Omega, \quad \theta_{\mid \Sigma}^{\varepsilon}=0,
\end{array}\right.
$$

where $f^{\varepsilon}, g^{\varepsilon}, u_{0}^{\varepsilon}$ and $\theta_{0}^{\varepsilon}$ are $C^{\infty}$ regularizations by convolution of $f, g, u_{0}$ and $\theta_{0}$, respectively. Let $\gamma$ be any number of the interval $(0,1)$.

Proposition 3.1. Under the assumption (6), for $\theta \in C_{\gamma, \gamma / 2}^{1,0}(\bar{Q})$, problem

$$
\left\{\begin{array}{l}
\partial_{t} u^{\varepsilon}-\triangle u^{\varepsilon}+m \eta_{\varepsilon}\left(u^{\varepsilon}-A \theta\right)-m \eta_{\varepsilon}\left(B \theta-u^{\varepsilon}\right)=f^{\varepsilon} \quad \text { in } Q \\
u^{\varepsilon}(0)=u_{0}^{\varepsilon} \text { on } \Omega, \quad u_{\mid \Sigma}^{\varepsilon}=0
\end{array}\right.
$$

has a unique solution $u^{\varepsilon} \in C_{\gamma, \gamma / 2}^{2,1}(\bar{Q})$. Moreover, there exist $C_{1}$ and $C_{2}$, positive constants, independent of $\varepsilon$ and $\theta$, such that

$$
\left\|u^{\varepsilon}\right\|_{C_{\gamma, \gamma / 2}^{2,1}(\bar{Q})} \leq C_{1}\left(\left\|f^{\varepsilon}\right\|_{C_{\gamma, \gamma / 2}^{0}(\bar{Q})}+m+\left\|u_{0}^{\varepsilon}\right\|_{C_{\gamma}^{2}(\bar{\Omega})}\right)
$$

and

$$
\left\|u^{\varepsilon}\right\|_{W_{p}^{2,1}(Q)} \leq C_{2}\left(\|f\|_{L^{p}(Q)}+m+\left\|u_{0}\right\|_{W_{p}^{2}(\Omega)}\right)
$$

Remark 3.2. We observe that the estimate (13) depends on $\varepsilon$ and the estimate (14) does not, as the sequences $\left\{f^{\varepsilon}\right\}_{\varepsilon}$ and $\left\{u_{0}^{\varepsilon}\right\}_{\varepsilon}$ converge to $f$ and $u_{0}$, respectively in $L^{p}(\Omega)$ and in $W_{p}^{2}(\Omega)$, but not necessarily in $C_{\gamma, \gamma / 2}^{0}(\bar{Q})$ and $C_{\gamma}^{2}(\bar{\Omega})$.

Proof. The operator $R$ defined by

$$
<R z, w>=\int_{\Omega}\left(\eta_{\varepsilon}(z-A \theta)-\eta_{\varepsilon}(B \theta-z)\right) w
$$

is monotone. In fact

$$
\begin{aligned}
<R z-R w, z-w>= & \int_{\Omega}\left(\eta_{\varepsilon}(z-A \theta)-\eta_{\varepsilon}(w-A \theta)\right)((z-A \theta)-(w-A \theta))+ \\
& \int_{\Omega}\left(\eta_{\varepsilon}(B \theta-z)-\eta_{\varepsilon}(B \theta-w)\right)((B \theta-z)-(B \theta-w)) \geq 0,
\end{aligned}
$$


as $\eta_{\varepsilon}$ is nondecreasing.

By a well known result (see [9]), problem $(12-\theta)$ has a unique solution $u^{\varepsilon} \in L^{2}\left(0, T ; H_{0}^{1}(\Omega)\right)$. But

$$
\left\{\begin{array}{l}
\partial_{t} u^{\varepsilon}-\triangle u^{\varepsilon}=F^{\varepsilon} \quad \text { in } Q \\
u^{\varepsilon}(0)=u_{0}^{\varepsilon} \text { on } \Omega, u_{\mid \Sigma}^{\varepsilon}=0,
\end{array}\right.
$$

where $F^{\varepsilon}=f^{\varepsilon}-m \eta_{\varepsilon}\left(u^{\varepsilon}-A \theta\right)+m \eta_{\varepsilon}\left(B \theta-u^{\varepsilon}\right)$ belongs to $L^{p}(Q)$, as $\eta_{\varepsilon}$ is a bounded function. So $u^{\varepsilon} \in W_{p}^{2,1}(Q)$ and, by [8, Theorem 9.1], we obtain the inequality (14).

On the other hand, $F^{\varepsilon}$ is a $C^{\infty}$ function. So, applying Hölder estimates (see [8, Theorem 5.2]) we get the inequality (13).

Proposition 3.3. Under the assumptions (6) and (7), the function $T: C_{\gamma, \gamma / 2}^{1,0}(\bar{Q}) \rightarrow C_{\gamma, \gamma / 2}^{1,0}(\bar{Q})$ such that, for $\theta \in C_{\gamma, \gamma / 2}^{1,0}(\bar{Q}), T(\theta)$ is the solution of problem $(12-\theta)$, is continuous with bounded range independently of $\varepsilon$.

Proof. To prove the continuity of $T$ we only need to prove that, if $\left(\theta_{n}\right)_{n \in \mathbb{N}}$ is a sequence convergent in $C_{\gamma, \gamma / 2}^{1,0}(\bar{Q})$ to $\theta$, then $\left(T\left(\theta_{n}\right)\right)_{n \in \mathbb{N}}$ admits a subsequence convergent to $T(\theta)$. Let $u_{n}^{\varepsilon}=T\left(\theta_{n}\right)$ and $u^{\varepsilon}=T(\theta)$ and denote $\chi_{n}^{\varepsilon}=-m \eta_{\varepsilon}\left(u_{n}^{\varepsilon}-A \theta_{n}\right)+m \eta_{\varepsilon}\left(B \theta_{n}-u_{n}^{\varepsilon}\right)$ and $\chi^{\varepsilon}=-m \eta_{\varepsilon}\left(u^{\varepsilon}-A \theta\right)+m \eta_{\varepsilon}\left(B \theta-u^{\varepsilon}\right)$. Then

$$
\left\{\begin{array}{l}
\partial_{t}\left(u_{n}^{\varepsilon}-u^{\varepsilon}\right)-\triangle\left(u_{n}^{\varepsilon}-u^{\varepsilon}\right)=\chi_{\varepsilon}^{n}-\chi_{\varepsilon} \text { in } Q \\
\left(u_{n}^{\varepsilon}-u^{\varepsilon}\right)(0)=0 \text { on } \Omega, \quad\left(u_{n}^{\varepsilon}-u^{\varepsilon}\right)_{\mid \Sigma}=0
\end{array}\right.
$$

and, if $Q_{t}=\Omega \times(0, t)$, for $t \in(0, T)$, we have

$$
\frac{1}{2} \int_{\Omega}\left(u_{n}^{\varepsilon}(t)-u^{\varepsilon}(t)\right)^{2}+\int_{Q_{t}}\left|\nabla\left(u_{n}^{\varepsilon}-u^{\varepsilon}\right)\right|^{2}=\int_{Q_{t}}\left(\chi_{n}^{\varepsilon}-\chi^{\varepsilon}\right)\left(u_{n}^{\varepsilon}-u^{\varepsilon}\right) .
$$

Note that we have the following equality

$$
\begin{aligned}
\left(\chi_{n}^{\varepsilon}-\chi^{\varepsilon}\right) & \left(u_{n}^{\varepsilon}-u^{\varepsilon}\right) \\
= & \left(m \eta_{\varepsilon}\left(u^{\varepsilon}-A \theta_{n}\right)-m \eta_{\varepsilon}\left(u_{n}^{\varepsilon}-A \theta_{n}\right)\right)\left(\left(u_{n}^{\varepsilon}-A \theta_{n}\right)-\left(u^{\varepsilon}-A \theta_{n}\right)\right) \\
& +\left(m \eta_{\varepsilon}\left(B \theta_{n}-u^{\varepsilon}\right)-m \eta_{\varepsilon}\left(B \theta_{n}-u_{n}^{\varepsilon}\right)\right)\left(\left(B \theta_{n}-u_{n}^{\varepsilon}\right)-\left(B \theta_{n}-u^{\varepsilon}\right)\right) \\
& +\left(m \eta_{\varepsilon}\left(B \theta_{n}-u^{\varepsilon}\right)-m \eta_{\varepsilon}\left(B \theta-u^{\varepsilon}\right)+m \eta_{\varepsilon}\left(u^{\varepsilon}-A \theta\right)-m \eta_{\varepsilon}\left(u^{\varepsilon}-A \theta_{n}\right)\right)\left(u_{n}^{\varepsilon}-u^{\varepsilon}\right),
\end{aligned}
$$

where the first and second parcels are nonpositive, as $\eta_{\varepsilon}$ is nondecreasing. So, denoting the third parcel by $\Upsilon_{n}^{\varepsilon}\left(u_{n}^{\varepsilon}-u^{\varepsilon}\right)$, we have

$$
\frac{1}{2} \int_{\Omega}\left(u_{n}^{\varepsilon}(t)-u^{\varepsilon}(t)\right)^{2}+\int_{Q_{t}}\left|\nabla\left(u_{n}^{\varepsilon}-u^{\varepsilon}\right)\right|^{2} \leq \int_{Q_{t}} \Upsilon_{n}^{\varepsilon}\left(u_{n}^{\varepsilon}-u^{\varepsilon}\right) .
$$

But $\left(\Upsilon_{n}^{\varepsilon}\right)_{n \in \mathbb{N}}$ converges to 0 in $L^{1}(Q)$, when $n \rightarrow \infty$, by the dominated convergence theorem, as by (7) $A, B$ are continuous and as $\eta_{\varepsilon}$ is continuous and bounded for $\varepsilon$ fixed. By $(16),\left(u_{n}^{\varepsilon}-u^{\varepsilon}\right)_{n \in \mathbb{N}}$ is bounded in $L^{\infty}(Q)$. Then

$$
\left|\int_{Q_{t}} \Upsilon_{n}^{\varepsilon}\left(u_{n}^{\varepsilon}-u^{\varepsilon}\right)\right| \leq\left\|\Upsilon_{n}^{\varepsilon}\right\|_{L^{1}(Q)}\left\|u_{n}^{\varepsilon}-u^{\varepsilon}\right\|_{L^{\infty}(Q)} \underset{n}{\longrightarrow} 0
$$

and so, by (17), $\left(u_{n}^{\varepsilon}-u\right)_{n \in \mathbb{N}}$ converges to 0 in $L^{\infty}\left(0, T ; L^{2}(\Omega)\right) \cap L^{2}\left(0, T ; H_{0}^{1}(\Omega)\right)$. On the other hand, from (16) we deduce that this sequence is bounded in $W_{p}^{2,1}(Q)$. As $W_{p}^{2,1}(Q)$ is compactly included in $C_{\gamma, \gamma / 2}^{1,0}(\bar{Q})$, this sequence admits a subsequence that converges in $C_{\gamma, \gamma / 2}^{1,0}(\bar{Q})$ to a function that, as $C_{\gamma, \gamma / 2}^{1,0}(\bar{Q}) \hookrightarrow$ $L^{\infty}\left(0, T ; H_{0}^{1}(\Omega)\right)$, is the null function.

The fact that the range of $T$ is bounded (it may depend on $\varepsilon$ ) is a consequence of the inequality (13). 
Proposition 3.4. Under the assumption (5), for $u \in C_{\gamma, \gamma / 2}^{1,0}(\bar{Q})$, problem

$$
\left\{\begin{array}{l}
\partial_{t} \theta-\triangle \theta=g^{\varepsilon}(u) \quad \text { in } Q \\
\theta(0)=\theta_{0}^{\varepsilon} \text { on } \Omega, \quad \theta_{\mid \Sigma}=0
\end{array}\right.
$$

has a unique solution $\theta^{\varepsilon}$ that belongs to $C_{\gamma, \gamma / 2}^{2,1}(\bar{Q})$. Moreover, there exists a positive constant $C$, independent of $\varepsilon$ and $u$, such that

$$
\left\|\theta^{\varepsilon}\right\|_{C_{\gamma, \gamma / 2}^{2,1}(\bar{Q})} \leq C\left(\left\|g^{\varepsilon}(u)\right\|_{C_{\gamma, \gamma / 2}^{0}(\bar{Q})}+\left\|\theta_{0}^{\varepsilon}\right\|_{C_{\gamma}^{2}(\bar{\Omega})}\right)
$$

Proof. As $g^{\varepsilon} \circ u \in C_{\gamma, \gamma / 2}^{1,0}(\bar{Q})$ the proof is an immediate consequence of [8, Theorem 5.2].

Proposition 3.5. Under the assumption (5), the function $S: C_{\gamma, \gamma / 2}^{1,0}(\bar{Q}) \rightarrow C_{\gamma, \gamma / 2}^{2,1}(\bar{Q})$ such that, for $u \in$ $C_{\gamma, \gamma / 2}^{1,0}(\bar{Q}), S(u)$ is the solution of problem $(18-u)$, is continuous. Moreover $S$ transforms bounded sets into bounded sets (depending on $\varepsilon$ ).

Proof. Let $\left(u_{n}\right)_{n \in \mathbb{N}}$ be a sequence convergent to a function $u$ in $C_{\gamma, \gamma / 2}^{1,0}(\bar{Q})$. Let $\theta_{n}^{\varepsilon}=S\left(u_{n}\right)$ and $\theta^{\varepsilon}=S(u)$. We have

$$
\left\{\begin{array}{l}
\partial_{t}\left(\theta_{n}^{\varepsilon}-\theta^{\varepsilon}\right)-\triangle\left(\theta_{n}^{\varepsilon}-\theta^{\varepsilon}\right)=g^{\varepsilon}\left(u_{n}\right)-g^{\varepsilon}(u) \text { in } Q \\
\left(\theta_{n}^{\varepsilon}-\theta^{\varepsilon}\right)(0)=0 \text { on } \Omega, \quad \theta_{\mid \Sigma}=0 .
\end{array}\right.
$$

and then, as in the previous proposition,

$$
\left\|\theta_{n}^{\varepsilon}-\theta^{\varepsilon}\right\|_{C_{\gamma, \gamma / 2}^{2,1}(\bar{Q})} \leq C\left\|g^{\varepsilon}\left(u_{n}\right)-g^{\varepsilon}(u)\right\|_{C_{\gamma, \gamma / 2}^{1,0}(\bar{Q})}
$$

As $\left\|g^{\varepsilon}\left(u_{n}\right)-g^{\varepsilon}(u)\right\|_{C_{\gamma, \gamma / 2}^{1,0}(\bar{Q})}^{\longrightarrow} \underset{n}{\longrightarrow} 0$, the continuity of $S$ is obvious. By inequality (19), $S$ transform bounded sets into bounded sets.

Theorem 3.6. Under the assumptions (5)-(7), the penalized problem (11) has a solution $\left(u^{\varepsilon}, \theta^{\varepsilon}\right) \in C_{\gamma, \gamma / 2}^{2,1}(\bar{Q}) \times$ $C_{\gamma, \gamma / 2}^{2,1}(\bar{Q})$.

Proof. Consider $\Phi=i \circ S \circ T$, where $i$ is the (compact) inclusion of $C_{\gamma, \gamma / 2}^{2,1}(\bar{Q})$ into $C_{\gamma, \gamma / 2}^{1,0}(\bar{Q})$. Using the previous propositions, we conclude that $\Phi: C_{\gamma, \gamma / 2}^{1,0}(\bar{Q}) \rightarrow C_{\gamma, \gamma / 2}^{1,0}(\bar{Q})$ is continuous and bounded. As $i$ is compact, $\Phi$ is also compact. So, by Schauder fixed point theorem, there exists a fixed point $\theta^{\varepsilon}$ of $\Phi$. Therefore, if $u^{\varepsilon}=T\left(\theta^{\varepsilon}\right)$, then $\left(u^{\varepsilon}, \theta^{\varepsilon}\right)$ is a solution of the system in the desired conditions.

\subsection{Existence of solution}

We are now in a condition to prove the existence of solution of problem (4)-(2).

We start by proving a result that will allow us to show that the solutions of the approximating problems converge, when $\varepsilon$ tends to zero, to a pair $(u, \theta)$ satisfying $u(t) \in \mathbb{K}_{A \theta(t)}^{B \theta(t)}$ for a.e. $t \in(0, T)$.

Proposition 3.7. Under the assumptions (5)-(9), for a sufficiently large choice of $m$, independent of $\varepsilon \in(0,1)$, the solution $\left(u^{\varepsilon}, \theta^{\varepsilon}\right)$ of problem (11) satisfies

$$
\left\|\left(u^{\varepsilon}-B \theta^{\varepsilon}-\varepsilon\right)^{+}\right\|_{L^{\infty}\left(0, T ; L^{2}(\Omega)\right)}^{2}+\left\|\nabla\left(u^{\varepsilon}-B \theta^{\varepsilon}-\varepsilon\right)^{+}\right\|_{L^{2}(Q)}^{2} \leq\left\|\left(u_{0}^{\varepsilon}-B \theta_{0}^{\varepsilon}-\varepsilon\right)^{+}\right\|_{L^{2}(\Omega)}^{2},
$$

and

$$
\left\|\left(u^{\varepsilon}-A \theta^{\varepsilon}+\varepsilon\right)^{-}\right\|_{L^{\infty}\left(0, T ; L^{2}(\Omega)\right)}^{2}+\left\|\nabla\left(u^{\varepsilon}-A \theta^{\varepsilon}+\varepsilon\right)^{-}\right\|_{L^{2}(Q)}^{2} \leq\left\|\left(u_{0}^{\varepsilon}-A \theta_{0}^{\varepsilon}+\varepsilon\right)^{-}\right\|_{L^{2}(\Omega)}^{2} .
$$


Proof. Multiply the first equation of problem (11) by $\left(u^{\varepsilon}-B \theta^{\varepsilon}-\varepsilon\right)^{+}$and integrate over $Q_{t}$, obtaining

$$
\begin{array}{r}
\int_{Q_{t}}\left(\partial_{t}\left(u^{\varepsilon}-B \theta^{\varepsilon}-\varepsilon\right)-\Delta\left(u^{\varepsilon}-B \theta^{\varepsilon}-\varepsilon\right)-f^{\varepsilon}\right)\left(u^{\varepsilon}-B \theta^{\varepsilon}-\varepsilon\right)^{+}+\int_{Q_{t}}\left(\partial_{t} B \theta^{\varepsilon}-\Delta B \theta^{\varepsilon}\right)\left(u^{\varepsilon}-B \theta^{\varepsilon}-\varepsilon\right)^{+} \\
+\int_{Q_{t}}\left(m \eta_{\varepsilon}\left(u^{\varepsilon}-A \theta^{\varepsilon}\right)-m \eta_{\varepsilon}\left(B \theta^{\varepsilon}-u^{\varepsilon}\right)\right)\left(u^{\varepsilon}-B \theta^{\varepsilon}-\varepsilon\right)^{+} \leq 0
\end{array}
$$

But

$$
\left(m \eta_{\varepsilon}\left(u^{\varepsilon}-A \theta^{\varepsilon}\right)-m \eta_{\varepsilon}\left(B \theta^{\varepsilon}-u^{\varepsilon}\right)\right)\left(u^{\varepsilon}-B \theta^{\varepsilon}-\varepsilon\right)^{+}=m\left(u^{\varepsilon}-B \theta^{\varepsilon}-\varepsilon\right)^{+},
$$

which implies that

$$
\begin{aligned}
\frac{1}{2} \int_{\Omega}\left(\left(u^{\varepsilon}(t)-B \theta^{\varepsilon}(t)-\varepsilon\right)^{+}\right)^{2}+\int_{Q_{t}}\left|\nabla\left(u^{\varepsilon}-B \theta^{\varepsilon}-\varepsilon\right)^{+}\right|^{2} \\
\quad+\int_{Q_{t}}\left(\partial_{t} B \theta^{\varepsilon}-\Delta B \theta^{\varepsilon}-f^{\varepsilon}+m\right)\left(u^{\varepsilon}-B \theta^{\varepsilon}-\varepsilon\right)^{+} \leq \frac{1}{2} \int_{\Omega}\left(\left(u_{0}^{\varepsilon}-B \theta_{0}^{\varepsilon}-\varepsilon\right)^{+}\right)^{2}
\end{aligned}
$$

By assumption (9) we have

$$
\partial_{t} B \theta^{\varepsilon}-\Delta B \theta^{\varepsilon}-f^{\varepsilon} \geq-c\left|\partial_{t} \theta^{\varepsilon}-\Delta \theta^{\varepsilon}\right|-\left\|f^{\varepsilon}\right\|_{L^{\infty}(Q)} \geq-c\left|g\left(u^{\varepsilon}\right)\right|-\|f\|_{L^{\infty}(Q)} .
$$

Recall that $W_{p}^{2,1}(Q) \subseteq L^{\infty}(Q)$. So, using (14), we have

$$
\left\|u^{\varepsilon}\right\|_{L^{\infty}(Q)} \leq c_{0} m+c_{1}
$$

being $c_{0}$ and $c_{1}$ positive constants independent of $\varepsilon$. But, using (8) we have

$$
c\left|g\left(u^{\varepsilon}\right)\right|+\|f\|_{L^{\infty}(Q)} \leq c\left(a_{0}\left|u^{\varepsilon}\right|^{r}+a_{1}\right) \leq c\left(\left(a_{0}\left(c_{0} m+c_{1}\right)^{r}+a_{1}\right)\right)+\|f\|_{L^{\infty}(Q)} \leq m,
$$

for $m$ large enough. So $\partial_{t} B \theta^{\varepsilon}-\Delta B \theta^{\varepsilon}+m \geq 0$ and going back to inequality (22), we conclude that

$$
\frac{1}{2} \int_{\Omega}\left(\left(u^{\varepsilon}(t)-B \theta^{\varepsilon}(t)-\varepsilon\right)^{+}\right)^{2}+\int_{Q_{t}}\left|\nabla\left(u^{\varepsilon}-B \theta^{\varepsilon}-\varepsilon\right)^{+}\right|^{2} \leq \frac{1}{2} \int_{\Omega}\left(\left(u_{0}^{\varepsilon}-B \theta_{0}^{\varepsilon}-\varepsilon\right)^{+}\right)^{2},
$$

from where we obtain (20). Reasoning similarly, we get the other inequality.

Proof. of Theorem 2.3 Let $\left(u^{\varepsilon}, \theta^{\varepsilon}\right)$ be the solution of the penalized problem given by Theorem 3.6, for a choice of $m$ suitable for Proposition 3.7. Recall that $m$ does not depend on $\varepsilon$ and $f \varepsilon \underset{\varepsilon \rightarrow 0}{\longrightarrow} f$ in $L^{p}(\Omega)$. By the inequality (14), $\left\{u^{\varepsilon}\right\}_{\varepsilon}$ is bounded in $W_{p}^{2,1}(Q)$ and so, for a subsequence, we have, when $\varepsilon \rightarrow 0$,

$$
u^{\varepsilon} \rightarrow u \text { in } W_{p}^{2,1}(Q) \text {-weak. }
$$

Due to the compact inclusion of $W_{p}^{2,1}(Q)$ in $C_{\gamma, \gamma / 2}^{1,0}(\bar{Q})$, for $0<\gamma<1-d / p$, we also have

$$
u^{\varepsilon} \rightarrow u \text { in } C_{\gamma, \gamma / 2}^{1,0}(\bar{Q})
$$

Let us call $N=\|u\|_{C(\bar{Q})}$. Then, using (24), there exists $\varepsilon_{0}>0$ such that, if $0<\varepsilon \leq \varepsilon_{0}$ then $\left\|u^{\varepsilon}\right\|_{C(\bar{Q})} \leq$ $N+1$. And so, as $g^{\varepsilon} \rightarrow g$ uniformly in the compact subsets of $\mathbb{R}$, then $\left|g^{\varepsilon}\left(u^{\varepsilon}\right)\right| \leq\left\|g^{\varepsilon}\right\|_{C([-N-1, N+1])} \leq$ $\|g\|_{C([-N-1, N+1])}+1$ and so the sequence $\left\{g^{\varepsilon}\left(u^{\varepsilon}\right)\right\}_{\varepsilon}$ is bounded by the constant function $\left(\|g\|_{C([-N-1, N+1])}+1\right)$ 
in $L^{r}(Q)$, for any $1<r<\infty$. As, for any $(x, t) \in Q$, we have $g^{\varepsilon}\left(u^{\varepsilon}(x, t)\right) \rightarrow g(u(x, t))$, when $\varepsilon \rightarrow 0$, by the dominated convergence theorem we conclude that, letting $\varepsilon \rightarrow 0$,

$$
g^{\varepsilon}\left(u^{\varepsilon}\right) \rightarrow g(u) \text { in } L^{q}(Q)
$$

The function $\theta^{\varepsilon}$ satisfies the inequality

$$
\left\|\theta^{\varepsilon}\right\|_{W_{q}^{2,1}(Q)} \leq C\left(\left\|g^{\varepsilon}\left(u^{\varepsilon}\right)\right\|_{L^{q}(Q)}+\left\|\theta_{0}\right\|_{W_{q}^{2}(\Omega)}\right)
$$

being $C$ a positive constant independent of $\varepsilon$. Using (25), we conclude that $\left\{\theta^{\varepsilon}\right\}_{\varepsilon}$ is bounded in $W_{q}^{2,1}(Q)$ and, at least for a subsequence, we also have, when $\varepsilon \rightarrow 0$,

$$
\theta^{\varepsilon} \rightarrow \theta \text { in } W_{q}^{2,1}(Q) \text {-weak, } \quad \theta^{\varepsilon} \rightarrow \theta \text { in } C_{\gamma, \gamma / 2}^{1,0}(\bar{Q}), 0<\gamma<1-d / q \text {. }
$$

Passing to the limit, when $\varepsilon \rightarrow 0$ in the two last equations of problem (11), we get

$$
\partial_{t} \theta-\Delta \theta=g(u) \text { in } \Omega, \quad \theta(0)=\theta_{0} \text { in } \Omega, \quad \theta_{\left.\right|_{\Sigma}}=0 .
$$

In order to prove that $(u, \theta)$ satisfies (4)-(2), let $t \in(0, T)$ and $v \in \mathbb{K}_{A \theta(t)}^{B \theta(t)}$. Consider $v^{\varepsilon}=\left(v \wedge B \theta^{\varepsilon}(t)\right) \vee$ $A \theta^{\varepsilon}(t)$ and note that $v^{\varepsilon}(t) \in \mathbb{K}_{A \theta^{\varepsilon}(t)}^{B \theta^{\varepsilon}(t)}$ and $v^{\varepsilon}(t) \underset{\varepsilon \rightarrow 0}{\longrightarrow} v(t)$ in $H^{1}(\Omega)$. Besides,

$$
\left(\eta_{\varepsilon}\left(u^{\varepsilon}-A \theta^{\varepsilon}\right)-\eta_{\varepsilon}\left(B \theta^{\varepsilon}-u^{\varepsilon}\right)\right)\left(v^{\varepsilon}-u^{\varepsilon}\right) \begin{cases}=0 & \text { if } A \theta^{\varepsilon} \leq u^{\varepsilon} \leq B \theta^{\varepsilon} \\ <0 & \text { otherwise. }\end{cases}
$$

So, from (11) and integrating by parts we obtain,

$$
\int_{\Omega} \partial_{t} u^{\varepsilon}(t)\left(v^{\varepsilon}-u^{\varepsilon}(t)\right)+\int_{\Omega} \nabla u^{\varepsilon}(t) \cdot \nabla\left(v^{\varepsilon}-u^{\varepsilon}(t)\right) \geq \int_{\Omega} f(t)\left(v^{\varepsilon}-u^{\varepsilon}(t)\right)
$$

and then

$$
\int_{\Omega} \partial_{t} u(t)(v-u(t))+\int_{\Omega} \nabla u(t) \cdot \nabla(v-u(t)) \geq \int_{\Omega} f(t)(v-u(t)) .
$$

As $u(0)=u_{0}$, the conclusion that $u$ solves (4) follows if we prove that $u(t) \in \mathbb{K}_{A \theta(t)}^{B \theta(t)}$. Letting $\varepsilon \rightarrow 0$ in inequalities (20) and (21) and using the fact that the operators $A$ and $B$ are continuous, we conclude that

$$
\left\|(u-B \theta)^{+}\right\|_{L^{\infty}\left(0, T ; L^{2}(\Omega)\right)}=0 \quad \text { and } \quad\left\|(u-A \theta)^{-}\right\|_{L^{\infty}\left(0, T ; L^{2}(\Omega)\right)}=0
$$

So,

$$
A \theta \leq u \leq B \theta \quad \text { a.e. in } Q
$$

Proof. of Corollary 2.4 We start by defining an interval $I$, depending only on the given data, such that the domain of $A$ and $B$ is $C(\bar{Q} ; I)$ and the solution $\theta$ of problem (2) has range contained in $I$. We remark that the functions $\varphi$ and $\psi$ of example 2.1 cannot have domain $\mathbb{R}$ because $\varphi, \psi$ are bounded, $\varphi<\psi, \varphi$ is convex and $\psi$ is concave.

Let $\left(u^{\varepsilon}, \theta^{\varepsilon}\right)$ be the solution of the penalized problem (11). By (14),

$$
\left\|u^{\varepsilon}\right\|_{W_{p}^{2,1}(Q)} \leq C_{2}\left(\|f\|_{L^{p}(Q)}+m+\left\|u_{0}\right\|_{W_{p}^{2}(\Omega)}\right)=N
$$


where $m=m\left(c, a_{0}, a_{1}\right)$ was fixed in (23). So, $N=N\left(p, Q, c, a_{0}, a_{1},\|f\|_{L^{p}(Q)},\left\|u_{0}\right\|_{W_{p}^{2}(\Omega)}\right)$.

By (26) and the previous estimate,

$$
\left\|\theta^{\varepsilon}\right\|_{W_{q}^{2,1}(Q)} \leq C\left(\left(\|g\|_{C([-N, N])}+1\right)|Q|^{\frac{1}{q}}+\left\|\theta_{0}\right\|_{W_{q}^{2}(\Omega)}\right)=M .
$$

Notice that

$$
M=M\left(p, q, Q, c, a_{0}, a_{1},\|f\|_{L^{p}(Q)},\left\|u_{0}\right\|_{W_{p}^{2}(\Omega)},\|g\|_{C([-N, N])},\left\|\theta_{0}\right\|_{W_{q}^{2}(\Omega)}\right) .
$$

The interval $I$ can be chosen as any interval that contains $[-M, M]$.

Now, we only need to show that the operators $A$ and $B$ satisfy $A<B, A 0 \leq 0 \leq B 0$, satisfy (9) and are continuous.

As $\varphi<\psi$ then $A \theta=\varphi(\theta)<\psi(\theta)=B \theta$, for any $\theta \in C(\bar{Q} ; I)$. And $A 0=\varphi(0) \leq 0 \leq \psi(0)=B 0$.

Easy calculations show that

$$
\partial_{t}(A \theta)-\Delta(A \theta)=\varphi^{\prime}(\theta)\left(\partial_{t} \theta-\Delta \theta\right)-\varphi^{\prime \prime}(\theta)|\nabla \theta|^{2} \leq D\left|\partial_{t} \theta-\Delta \theta\right|,
$$

as, by assumption, $\left|\varphi^{\prime}\right|$ is bounded and $\varphi^{\prime \prime} \geq 0$. Given $\theta_{1}, \theta_{2} \in C(\bar{Q} ; I)$ and $(x, t) \in \bar{Q}$, we have

$$
\left|A \theta_{1}(x, t)-A \theta_{2}(x, t)\right|=\left|\varphi\left(\theta_{1}(x, t)\right)-\varphi\left(\theta_{2}(x, t)\left|\leq\left\|\varphi^{\prime}\right\|_{L^{\infty}(I)}\right| \mid \theta_{1}(x, t)-\theta_{2}(x, t)\right)\right|
$$

and so

$$
\left\|A \theta_{1}-A \theta_{2}\right\|_{C(\bar{Q} ; \mathbb{R})} \leq\left\|\varphi^{\prime}\right\|_{L^{\infty}(I)} \mid\left\|\theta_{1}-\theta_{2}\right\|_{C(\bar{Q} ; I)},
$$

proving that $A$ is continuous. The corresponding proofs for $B$ are similar.

The conclusion of the corollary follows from Theorem 2.3.

\section{The gradient constraint system}

This section is dedicated to the proof of Theorem 2.5. We will also use the approximating problem (11) defined in Section 3. We will choose $m$ satisfying (36).

We need to present some results concerning the special type of obstacles we are considering.

We start by recalling the definition of the operators $A$ and $B$ of Example 2.2:

$$
A \theta(t)=\bigwedge_{v \in \mathbb{K}_{F(\theta(t))}^{\nabla}} v, \quad \text { and } \quad B \theta(t)=\bigvee_{v \in \mathbb{K}_{F(\theta(t))}^{\nabla}} v,
$$

where $\mathbb{K}_{F(\theta(t))}^{\nabla}$ is defined in (3). In this section we are assuming that $F \in C^{2}(\mathbb{R})$ and satisfies $0<F_{*} \leq F \leq$ $F^{*}$.

It is known (see [10, Theorem 5.1]) that

$$
A \theta(t)=-L_{t}(x, \partial \Omega) \quad \text { and } \quad B \theta(t)=L_{t}(x, \partial \Omega),
$$

where $L_{t}$ is the metric defined in $\bar{\Omega}$ by

$$
L_{t}(x, z)=\inf \left\{\int_{0}^{\delta} F(\theta(\xi(s), t)) d s: \delta>0, \xi:[0, \delta] \rightarrow \bar{\Omega} \text { smooth, } \xi(0)=x, \xi(\delta)=z,\left\|\xi^{\prime}\right\| \leq 1\right\} .
$$

In the special case $F \equiv 1$, the distance defined above is called the geodesic distance. We will denote it by $\bar{d}$. 
Lemma 4.1. For $x, z \in \bar{\Omega}$,

$$
\begin{aligned}
& L_{t}(x, z)=\inf \left\{\int_{0}^{\delta} F(\theta(\xi(s), t)) d s: 0<\delta \leq \frac{F^{*}}{F_{*}} \bar{\delta}(\Omega), \xi:[0, \delta] \rightarrow \bar{\Omega} \text { smooth },\right. \\
& \left.\xi(0)=x, \xi(\delta)=z,\left\|\xi^{\prime}\right\| \leq 1\right\},
\end{aligned}
$$

where $\bar{\delta}(\Omega)$ denotes the diameter of $\Omega$ for the geodesic distance $\bar{d}$.

Proof. Let

$$
\mathcal{A}_{x, z}=\left\{\xi:[0, \delta] \rightarrow \bar{\Omega} \text { smooth }, \delta>0, \xi(0)=x, \xi(\delta)=z,\left\|\xi^{\prime}\right\| \leq 1\right\}
$$

As, by assumption, the function $F$ satisfies $F_{*} \leq F \leq F^{*}$, given $\xi:\left[0, \delta_{\xi}\right] \rightarrow \bar{\Omega}$ in $\mathcal{A}_{x, z}$, we have

$$
F_{*} \int_{0}^{\delta_{\xi}} 1 d s \leq \int_{0}^{\delta_{\xi}} F(\theta(\xi(s), t)) d s \leq F^{*} \int_{0}^{\delta_{\xi}} 1 d s .
$$

Applying infimum, when $\xi \in \mathcal{A}_{x, z}$ to the inequalities above, we get

$$
F_{*} \bar{d}(x, z) \leq L_{t}(x, z) \leq F^{*} \bar{d}(x, z)
$$

If there exists a smooth path $\xi:[0, \delta] \rightarrow \bar{\Omega}$ in $\mathcal{A}_{x, z}$ such that $\delta>\frac{F^{*}}{F_{*}} \bar{\delta}(\Omega)$ then,

$$
\int_{0}^{\delta} F(\theta(\xi(s), t)) d s \geq F_{*} \int_{0}^{\delta} 1 d s=F_{*} \delta>F^{*} \bar{\delta}(\Omega) \geq F^{*} \bar{d}(x, z) \geq L_{t}(x, z) .
$$

This means that these paths are irrelevant to the computation of $L_{t}(x, z)$.

Proposition 4.2. Let $F \in C^{2}(\mathbb{R}), 0<F_{*} \leq F \leq F^{*}$ and $\theta \in L^{\infty}\left(0, T ; C^{2}(\bar{\Omega})\right) \cap W_{\infty}^{1}\left(0, T ; L^{\infty}(\Omega)\right)$. Then the functions $A \theta$ and $B \theta$ defined in (27) satisfy

$$
\begin{aligned}
& A \theta, B \theta \in W_{\infty}^{1}(Q), \\
& \text { for a.e. } t \in I \forall x \in \bar{\Omega} \quad|\nabla A \theta(x, t)|=|\nabla B \theta(x, t)|=F(\theta(x, t)), \\
& \exists c>0: \quad-c \leq \Delta A \theta \quad \text { and } \quad \Delta B \theta \leq c \quad \text { in } L^{\infty}\left(0, T ; \mathcal{D}^{\prime}(\Omega)\right) .
\end{aligned}
$$

Proof. The proof of this proposition can be found in [14, Proposition 3.2], for a given gradient constraint belonging to $L^{\infty}\left(0, T ; C^{2}(\bar{\Omega})\right) \cap W_{\infty}^{1}\left(0, T ; L^{\infty}(\Omega)\right)$ instead of $F(\theta)$. The only property that requires a detailed proof here is that $\partial_{t}(A \theta)$ and $\partial_{t}(B \theta)$ belong to $L^{\infty}(Q)$. The proof will be done only for $B \theta$.

For $(x, t) \in \bar{Q}$ and $h>0$, consider, using Lemma 4.1, $z \in \partial \Omega$, a smooth path $\xi:[0, \delta] \rightarrow \bar{\Omega}$ from $x$ to $z$ in $\Omega$, with $\delta \leq \frac{F^{*}}{F_{*}} \bar{\delta}(\Omega)$, such that $\left\|\xi^{\prime}\right\| \leq 1$ and $B \theta(x, t) \geq \int_{0}^{\delta} F(\theta(\xi(s), t)) d s-h$. Then

$$
\begin{aligned}
\frac{B \theta(x, t+h)-B \theta(x, t)}{h} & \leq \int_{0}^{\delta} \frac{F(\theta(\xi(s), t+h))-F(\theta(\xi(s), t))}{h} d s+1 \\
& =\int_{0}^{\delta}\left(\partial_{t}(F \theta)(\xi(s), \eta(h))\right) d s+1 \quad \text { with } t<\eta(h)<t+h \\
& \leq\left\|F^{\prime}\right\|_{L^{\infty}(-M, M)}\left\|\partial_{t} \theta\right\|_{L^{\infty}(Q)} \delta+1 \\
& \leq\left\|F^{\prime}\right\|_{L^{\infty}(-M, M)}\left\|\partial_{t} \theta\right\|_{L^{\infty}(Q)} \frac{F^{*}}{F_{*}} \bar{\delta}(\Omega)+1,
\end{aligned}
$$

where $M=\|\theta\|_{L^{\infty}(Q)}$. 
On the other hand, by the definition of infimum,

$$
\begin{array}{r}
B \theta(x, t+h)-B \theta(x, t) \geq \inf \left\{\int_{0}^{\delta}(F(\theta(\xi(s), t+h))-F(\theta(\xi(s), t))) d s: 0<\delta \leq \frac{F^{*}}{F_{*}} \bar{\delta}(\Omega)\right. \\
\left.\xi:[0, \delta] \rightarrow \bar{\Omega} \text { smooth, } \xi(0)=x, \xi(\delta) \in \partial \Omega,\left\|\xi^{\prime}\right\| \leq 1\right\} .
\end{array}
$$

As above, $F(\theta(\xi(s), t+h))-F(\theta(\xi(s), t)) \geq-\left\|F^{\prime}\right\|_{L^{\infty}(-M, M)}\left\|\partial_{t} \theta\right\|_{L^{\infty}(Q)} \frac{F^{*}}{F_{*}} \bar{\delta}(\Omega) h$ and then

$$
\frac{B \theta(x, t+h)-B \theta(x, t)}{h} \geq-\left\|F^{\prime}\right\|_{L^{\infty}(-M, M)}\left\|\partial_{t} \theta\right\|_{L^{\infty}(Q)} \frac{F^{*}}{F_{*}} \bar{\delta}(\Omega) .
$$

So $\partial_{t}(B \theta) \in L^{\infty}(Q)$.

By [10, Theorem 5.1], we know that, for a.e. $t \in(0, T), B \theta(t) \in W_{\infty}^{1}(\Omega)$ and that (30) is verified. So $B \theta \in L^{\infty}\left(0, T ; W_{\infty}^{1}(\Omega)\right)$.

By [10, Theorem 8.2], we know that, defining $\Omega_{\delta}=\{x \in \Omega: d(x, \partial \Omega) \geq \delta\}$ for $\delta>0$, then

$$
\exists \delta_{0}>0 \text { for a.e. } t \in(0, T) \quad B \theta(t) \in C^{2}\left(\bar{\Omega} \backslash \Omega_{\delta_{0}}\right) .
$$

And, using again [10, Theorem 5.1], we also have that

$$
\forall \delta>0 \exists C_{\delta}>0 \quad \text { for a.e. } t \in(0, T): \quad \Delta B \theta(t) \leq C_{\delta} \quad \text { in } \mathcal{D}^{\prime}\left(\Omega_{\delta}\right) .
$$

So, fixing $\delta=\delta_{0}$ in (34), (31) follows for $B \theta$ from (33) and (34).

Proof. of Theorem 2.5 We start by noticing that, with similar arguments as in the proof of Proposition 4.2, it is easy to verify that the operators $A$ and $B$ are Lipschitz. Besides, using (28) and (29), it is obvious that $A<B$ and $A 0<0<B 0$.

By Theorem 3.6, problem (11) has a solution $\left(u^{\varepsilon}, \theta^{\varepsilon}\right) \in C_{\gamma, \gamma / 2}^{2,1}(\bar{Q}) \times C_{\gamma, \gamma / 2}^{2,1}(\bar{Q})$, for any $0<\gamma \leq 1$. By inequality (14), there exist positive constants $c_{1}$ and $c_{2}$, independent of $\varepsilon$ and $m$, such that

$$
\left\|u^{\varepsilon}\right\|_{W_{p}^{2,1}(Q)} \leq c_{1} m+c_{2}
$$

So, $\left\{u^{\varepsilon}\right\}_{\varepsilon}$ is bounded in $W_{p}^{2,1}(Q)$, but this bound depends on $m$. As $C_{\alpha, \alpha / 2}^{1,0}(\bar{Q}) \hookrightarrow W_{p}^{2,1}(Q)$, we also have

$$
\left\|u^{\varepsilon}\right\|_{C_{\alpha, \alpha / 2}^{1,0}(\bar{Q})} \leq c_{1}^{\prime} m+c_{2}^{\prime}
$$

for positive constants $c_{1}^{\prime}$ and $c_{2}^{\prime}$.

The function $\theta_{\varepsilon}$ satisfies the inequality (19) with $g^{\varepsilon}$ substituted by $g$, as $g \in C_{\beta}^{0}(\mathbb{R})$ and $\gamma=\alpha \beta$.

Recalling (10), we get

$$
\begin{aligned}
\left\|g\left(u^{\varepsilon}\right)\right\|_{C_{\alpha \beta, \alpha \beta / 2}^{0}(\bar{Q})} & =\left\|g\left(u^{\varepsilon}\right)\right\|_{C^{0}(\bar{Q})}+M\left\|u^{\varepsilon}\right\|_{C_{\alpha, \alpha / 2}^{0}(\bar{Q})}^{\beta} \\
& \leq M\left\|u^{\varepsilon}\right\|_{C^{0}(\bar{Q})}^{\beta}+g(0)+M\left(c_{1} m+c_{2}\right)^{\beta} \\
& \leq d_{1} m^{\beta}+d_{2},
\end{aligned}
$$

being $d_{1}$ and $d_{2}$ positive constants independent of $\varepsilon$ and $m$.

Define

$$
\bar{\varphi}=B \theta^{\varepsilon}+\varepsilon \quad \text { and } \quad \underline{\varphi}=A \theta^{\varepsilon}-\varepsilon
$$


Observe that, using (19) and (35), we have

$$
\left\|\partial_{t} \theta^{\varepsilon}\right\|_{\left.L^{\infty}(Q)\right)} \leq C\left\|\theta^{\varepsilon}\right\|_{C_{\alpha \beta, \alpha \beta / 2}^{2,1}(\bar{Q})} \leq d_{1} m^{\beta}+d_{2},
$$

$d_{1}$ and $d_{2}$ positive constants.

Applying (31) and (32) and the previous inequality, we get

$\partial_{t} \bar{\varphi}-\Delta \bar{\varphi} \geq-\left\|F^{\prime}\right\|_{L^{\infty}(-M, M)}\left\|\partial_{t} \theta^{\varepsilon}\right\|_{L^{\infty}(Q)} \frac{F^{*}}{F_{*}} \bar{\delta}(\Omega)-c \geq-\left\|F^{\prime}\right\|_{L^{\infty}(-M, M)} \frac{F^{*}}{F_{*}}\left(d_{1} m^{\beta}+d_{2}\right)^{\beta} \bar{\delta}(\Omega) M-c$ and so

$$
\begin{aligned}
\partial_{t} \bar{\varphi}-\Delta \bar{\varphi}+m \eta_{\varepsilon}\left(\bar{\varphi}-A \theta^{\varepsilon}\right)-m \eta_{\varepsilon}\left(B \theta^{\varepsilon}-\bar{\varphi}\right) & \\
& \geq m-\left\|F^{\prime}\right\|_{L^{\infty}(-M, M)} \frac{F^{*}}{F_{*}}\left(d_{1} m^{\beta}+d_{2}\right)^{\beta} \bar{\delta}(\Omega) M-c \geq\|f\|_{L^{\infty}(Q)}
\end{aligned}
$$

as long as we choose

$$
m \geq\left\|F^{\prime}\right\|_{L^{\infty}(-M, M)} \frac{F^{*}}{F_{*}}\left(d_{1} m^{\beta}+d_{2}\right)^{\beta} \bar{\delta}(\Omega) M+c+\|f\|_{L^{\infty}(Q)} .
$$

So $\bar{\varphi}$ is a supersolution of the first equation of problem (11), as $\bar{\varphi}=\varepsilon>0$ on $\partial \Omega \times(0, T)$ and we also have $\bar{\varphi}(0) \geq u^{\varepsilon}(0)$ (recall the definition of $B \theta$ given in (27)). As the operator $R$, defined in (15), is monotone, we immediately have $u^{\varepsilon} \leq B \theta^{\varepsilon}+\varepsilon$.

To conclude that problem (4)-(2) has a solution, it is enough to follow the steps of the proof of Corollary 2.4 , with the natural adaptation of the spaces involved.

We are going to prove that the solution $(u, \theta)$ of problem (4)-(2) also solves the problem (1)-(2) if (i)-(iv) of this theorem are satisfied. For this purpose it is enough to show that $|\nabla u| \leq F \circ \theta$ a.e. in $Q$.

We start by differentiating the first equation of problem (11) with respect to $x_{k}$, multiply this last equation by $\partial_{x_{k}} u^{\varepsilon}$ and sum over $k$. Calling $v=\left|\nabla u^{\varepsilon}\right|^{2}$, as $\frac{1}{2} \partial_{t} v=\partial_{x_{k}} u^{\varepsilon} \partial_{x_{k}} \partial_{t} u^{\varepsilon}$ and $\frac{1}{2} \Delta v=\left(\partial_{x_{i}} \partial_{x_{k}} u^{\varepsilon}\right)^{2}+$ $\partial_{x_{k}} u^{\varepsilon} \Delta \partial_{x_{k}} u^{\varepsilon}$, we get

$$
\partial_{t} v-\Delta v+\frac{2}{\varepsilon}\left(v-\nabla \tilde{z} \cdot \nabla u^{\varepsilon}\right) \leq 0
$$

where

$$
\tilde{z}= \begin{cases}B \theta^{\varepsilon} & \text { if } u^{\varepsilon}>B \theta^{\varepsilon} \\ u^{\varepsilon} & \text { if } A \theta^{\varepsilon} \leq u^{\varepsilon} \leq B \theta^{\varepsilon} \\ A \theta^{\varepsilon} & \text { if } u^{\varepsilon}<A \theta^{\varepsilon}\end{cases}
$$

Multiplying Eq. (37) by $\left(v-F^{2}\left(\theta^{\varepsilon}\right)\right)^{+}$and integrating over $Q_{t}=\Omega \times(0, t)$, we obtain

$$
\begin{aligned}
\int_{Q_{t}} \partial_{t}\left(v-F^{2}\left(\theta^{\varepsilon}\right)\right)^{+}\left(v-F^{2}\left(\theta^{\varepsilon}\right)\right)^{+}+\int_{Q_{t}}\left|\nabla\left(v-F^{2}\left(\theta^{\varepsilon}\right)\right)^{+}\right|^{2} \\
+\frac{2}{\varepsilon} \int_{Q_{t}}\left(v-\nabla \tilde{z} \cdot \nabla u^{\varepsilon}\right)\left(v-F^{2}\left(\theta^{\varepsilon}\right)\right)^{+} \leq \int_{Q_{t}}\left(\partial_{t} F^{2}\left(\theta^{\varepsilon}\right)-\Delta F^{2}\left(\theta^{\varepsilon}\right)\right)\left(v-F^{2}\left(\theta^{\varepsilon}\right)\right)^{+}
\end{aligned}
$$

But

$$
v-\nabla \tilde{z} \cdot \nabla u^{\varepsilon} \leq v^{\frac{1}{2}}\left(v^{\frac{1}{2}}-|\nabla \tilde{z}|\right)= \begin{cases}v^{\frac{1}{2}}\left(v^{\frac{1}{2}}-\left|\nabla B \theta^{\varepsilon}\right|\right) & \text { if } u^{\varepsilon}>B \theta^{\varepsilon}, \\ 0 & \text { if } A \theta^{\varepsilon} \geq u^{\varepsilon} \leq B \theta^{\varepsilon} \\ v^{\frac{1}{2}}\left(v^{\frac{1}{2}}-\left|\nabla A \theta^{\varepsilon}\right|\right) & \text { if } A B \theta^{\varepsilon}<u^{\varepsilon},\end{cases}
$$


By (30) of Proposition 4.2, $\left|\nabla A \theta^{\varepsilon}\right|=\left|\nabla B \theta^{\varepsilon}\right|=F\left(\theta^{\varepsilon}\right)$ and so $\left(v-\nabla \tilde{z} \cdot \nabla u^{\varepsilon}\right)\left(v-F^{2}\left(\theta^{\varepsilon}\right)\right)^{+} \geq 0$.

On the other hand, if $G=F^{2}$, we have

$$
\partial_{t} G\left(\theta^{\varepsilon}\right)-\Delta G\left(\theta^{\varepsilon}\right)=G^{\prime}\left(\theta^{\varepsilon}\right)\left(\partial_{t} \theta^{\varepsilon}-\Delta \theta^{\varepsilon}\right)-G^{\prime \prime}(\theta)\left|\nabla \theta^{\varepsilon}\right|^{2}=G^{\prime}\left(\theta^{\varepsilon}\right) g\left(u^{\varepsilon}\right)-G^{\prime \prime}(\theta)\left|\nabla \theta^{\varepsilon}\right|^{2} \leq 0,
$$

by the assumptions (ii) and (iii).

Going back to inequality (38), we obtain

$$
\begin{aligned}
\frac{1}{2} \int_{\Omega}\left|\left(v(t)-F^{2}\left(\theta^{\varepsilon}(t)\right)\right)^{+}\right|^{2} & +\int_{Q_{t}}\left|\nabla\left(v-F^{2}\left(\theta^{\varepsilon}\right)\right)^{+}\right|^{2} \\
& \leq \int_{Q_{t}}\left(\partial_{t} F^{2}\left(\theta^{\varepsilon}\right)-\Delta F^{2}\left(\theta^{\varepsilon}\right)\right)\left(v-F^{2}\left(\theta^{\varepsilon}\right)\right)^{+}+\frac{1}{2} \int_{\Omega}\left|\left(v(0)^{2}-F^{2}\left(\theta_{0}^{\varepsilon}\right)\right)^{+}\right|^{2} .
\end{aligned}
$$

Recalling that $v=\left|\nabla u_{0}^{\varepsilon}\right|^{2}$ and the inequality (39), we get

$$
\frac{1}{2} \int_{\Omega}\left|\left(v(t)-F^{2}\left(\theta^{\varepsilon}(t)\right)\right)^{+}\right|^{2}+\int_{Q_{t}}\left|\nabla\left(v-F^{2}\left(\theta^{\varepsilon}\right)\right)^{+}\right|^{2} \leq \frac{1}{2} \int_{\Omega}\left|\left(\left|\nabla u_{0}^{\varepsilon}\right|^{2}-F^{2}\left(\theta_{0}^{\varepsilon}\right)\right)^{+}\right|^{2}
$$

and, passing to the limit in $\varepsilon$,

$$
\frac{1}{2} \int_{\Omega}\left|\left(|\nabla u(t)|^{2}-F^{2}(\theta(t))\right)^{+}\right|^{2}+\int_{Q_{t}}\left|\nabla\left(|\nabla u|^{2}-F^{2}(\theta)\right)^{+}\right|^{2} \leq 0
$$

and so

$$
|\nabla u| \leq F(\theta) \text { a.e. in } Q .
$$

Finally, if $(u, \theta)$ is a solution of problem (4)-(2), we have $u(t) \in \mathbb{K}_{F(\theta(t))}^{\nabla}$ and, as $\mathbb{K}_{F(\theta(t))}^{\nabla} \subset \mathbb{K}_{A \theta(t)}^{B \theta(t)}$, then $(u, \theta)$ also solves problem (1)-(2), concluding the proof.

\section{Acknowledgements}

This research was partially supported by CMAT - Centro de Matemática da Universidade do Minho, financed by the Strategic Project PEst-OE/MAT/UI0013/2014.

\section{References}

[1] A. Azevedo and L. Santos, A diffusion problem with gradient constraint depending on the temperature, Adv. Math. Sci. Appl. 20 (1) (2010), 153-168.

[2] Brezis, H., Multiplicateur de Lagrange en torsion elasto-plastique, Arch. Rational Mech. Anal. 49 (1972), 32-40.

[3] H. Brezis, L. Caffarelli and A. Friedman, Reinforcement problems for elliptic equations and variational inequalities, Ann. Mat. Pura Appl. 123 (4) (1980), 219-246.

[4] L. A. Caffarelli and A. Friedman, Reinforcement problems in elastoplasticity, Rocky Mountain J. Math. 10 (1980), 155-184.

[5] R. Kano, Applications of abstract parabolic quasi-variational inequalities to obstacle problems, "Nonlocal and abstract parabolic equations and their applications", Piotr Mucha, Marek Niezgódka and Piotr Rybka (eds.), Banach Center Publ., Polish Acad. Sci. Inst. Math., Warsaw, 86 2009, 163-174. 
[6] N. Kenmochi, Parabolic quasi-variational diffusion problems with gradient constraints, Discrete Contin. Dyn. Syst. Ser. S 6 (2013), no. 2, 423-438.

[7] M. Kunze and J. F. Rodrigues, An elliptic quasi-variational inequality with gradient constraints and some of its applications, Math. Methods Appl. Sci. 2310 (2000), 897-908.

[8] O. A. Ladyženskaja, V. A. Solonnikov, and N. N. Ural'ceva, Linear and Quasilinear Equations of Parabolic Type, Translations of Mathematical Monographs 23, AMS, 1968.

[9] J.-L. Lions, Quelques méthodes de résolution des problèmes aux limites non linéaires, Dunod, 1969.

[10] P.-L. Lions, Generalized solutions of Hamilton-Jacobi equations, Pitman (Advanced Publishing Program), 1982.

[11] L. Prigozhin, On the Bean critical-state model in superconductivity, European J. Appl. Math. 7 (3) (1996), 237-247.

[12] L. Prigozhin, Variational model of sandpile growth, European J. Appl. Math. 7 (3) (1996), 225-235.

[13] J. F. Rodrigues and L. Santos, A parabolic quasi-variational inequality arising in a superconductivity model, Ann. Scuola Norm. Sup. Pisa Cl. Sci. (4) 29 (2000), 153-169.

[14] L. Santos, Variational problems with non-constant gradient constraints, Port. Math. (N.S.), 59 (2) (2002), 205-248.

Assis Azevedo

Centre of Mathematics

University of Minho

Campus de Gualtar

4710-057 Braga

Portugal

assis@math.uminho.pt
Lisa Santos

Centre of Mathematics

University of Minho

Campus de Gualtar

4710-057 Braga

Portugal

lisa@math.uminho.pt 